\title{
Applicability of new radio technologies for advanced UMTS networks
}

\author{
A. Dekorsy, M. Schacht, S. Brueck, and G. Fischer \\ Lucent Technologies Networks System GmbH, Thurn-und-Taxis-Strasse 10, 90411 Nuremberg, Germany
}

\begin{abstract}
An expanded effort is under the way to support the evolution of UMTS (Universal Mobile Telecommunication System). Apart from delivering high data rates, future UMTS releases will also require to provide high network performance in terms of system capacity, low radiated power, and high coverage. Well promising performance-enhancing technologies are smart antennas as well as multiuser detection. Although these new radio technologies have recently been subject to intense research, main UMTS network integration aspects with their specific constraints have been neglected in many cases. Especially the interaction with UMTS radio resource control being required to meet Quality of Service $(\mathrm{QoS})$ constraints has to be included to assess the applicability of these technologies for UMTS.

In this paper, we study the interaction of beamforming concepts as well as multiuser detection with load and power control. We also work out UMTS specific constraints like signal-to-interference-plus-noise ratio (SINR) operating points, pilot power pollution or channel estimation, all strongly limiting network performance. Results are shown for capacity gains and power reduction for all beamforming concepts of interest as well as linear multiuser detection schemes. The results show that fix as well as user-specific beamforming significantly improves network performance gains in downlink. In uplink multiuser detection indicates fairly modest system capacity gains, while it reduces tremendously mobile station power.
\end{abstract}

\section{Introduction}

Future wireless mobile communication systems like the upcoming 3rd generation mobile system UMTS (Universal Mobile Telecommunication System) will be required to provide significant improvements in network performance. Rather than solely increasing the number of servable users, network improvement comprises also a reduced radiated power cor-

Correspondence to: A. Dekorsy

(dekorsy@lucent.com) responding to an increased coverage. Thus, CDMA (code division multiple access) based future communication systems have to balance the trade-off between system capacity, coverage and emission. In the last few years several new transceiver techniques and radio resource control strategies have been developed to cope for that challenge. Some major and well promising techniques being foreseen for UMTS application are (1) smart antennas and (2) multiuser detection. Several smart antennas like fixed beamforming and userspecific beamforming are of high interest for UMTS 3GPP (2002). While straight forward solutions exist for the uplink Godara (2002); Farrokhi et al. (1998), the downlink direction is much more challenging for UMTS, mainly caused by unknown channel profiles at the base stations as well as powerful pilot transmission Farrokhi et al. (1998); Schacht et al. (2003). Both fixed and user-specific beamforming lead to a performance increase by spatially suppressing inter/intracell interference. A further approach to cope with high intracell interference, solely for the uplink, is multiuser detection Verdu (1998). In UMTS, the user are generally separated in a code division manner that offers to apply more sophisticated receivers solutions like simple single user matched-filtering $(\mathrm{SUMF})^{1}$. In this paper we focus on Minimum Mean Square Error (MMSE) multiuser detection.

Recently, smart antennas as well as multiuser detection have been subject to intense research, but main network integration aspects with specific constraints have been often missed. As an example, the impact on radio resource control strategies has been rarely investigated up to now. Radio resource control is required since it is of utmost importance in UMTS to keep the air interface load under predefined thresholds to guarantee QoS requirements of already established calls Holma and Toskala (2000); Mueckenheim and Bernhard (2001). Thus, resource control strategies like power or load control has a strong impact on network performance and has to be included for performance assessments.

\footnotetext{
${ }^{1}$ Note that Rake detection represents single-user matched filtering. In this paper both terms are used in the same manner.
} 
The main objective of this article is to discuss UMTS network enhancements by applying smart antennas in downlink and multiuser detection in uplink direction. We depict concepts that are practicable for UMTS application and we gain an insight in their performance subject to UMTS constraints. Note that we restrict our investigations on UMTS dedicated channels only, but we also cover packet data transmission over that channels as well as mixed services. We present relative gain figures in terms of system capacity gain and emission reduction. Smart antennas for downlink beamforming are shown to deliver significantly higher network performance in terms of capacity as well as power reduction. With multiuser detection the performance increase is ambivalent. We observe moderate capacity gains only, but significant power reduction or coverage gains, respectively.

The paper is structured as follows. Section 2 deals with the interaction of beamforming and linear multiuser detection with power control and it introduces UMTS load control. In Sect. 3 we illuminate the beamforming concepts and give detailed system level results. Next, in Sect. 4 we describe multiuser detection for UMTS and we specially focus on the signal-to-interference-plus-noise ratio (SINR) operating points. Detailed network simulations are shown. Finally, Sect. 6 summarizes main aspects.

\section{Power- and load control}

\subsection{Power-control}

The enhancement of UMTS networks by advanced technologies like downlink beamforming or multiuser detection in the uplink requires investigating the interaction of these more sophisticated techniques with given radio resource management algorithms. A general view on power control can be found in the fundamental work by Yates (1995). A more detailed description on combining linear multiuser detection with power control for uplink transmission is reported in Ulukus and Yates (1997). In context with downlink beamforming, Farrokhi et al. (1998) introduced an iterative algorithm that converges to the optimum solution of power control and beamforming by utilizing a virtual uplink. Thus, the more sophisticated problem of downlink beamforming can be solved by leveraging on iterative algorithms known for uplink transmission.

Both, linear multiuser detection as well as beamforming can be interpreted as linear pre-filtering of received user signals. While beamforming acts in space domain, linear multiuser detection acts in the code domain. Thus, it is possible to set up a generic view on combining either beamforming or multiuser detection with power control. In the following, we summarize main aspects for the combination of linear prefiltering with power control being described in detail in the publications mentioned above. We restrict the description to uplink transmission, since the optimum downlink beamforming can be described by an virtual uplink Farrokhi et al. (1998).
The aim of jointly optimizing power control and linear pre-filtering the mixture of asynchronous received signals is to assign users with transmitted power levels so as to minimize the total transmitted power while ensuring a certain quality of service. We define here the quality of service (QoS) in terms of a signal-to-interference-plus-noise ratio (SINR) per user measured at the output of the linear filter. For notational convenience, we indicate the active users by $i=1, \ldots, K$ for the remainder of the paper, e.g. the user powers are denoted by $p_{i}$ with $i=1, \ldots, K$ and vector $\boldsymbol{p}=\left(p_{1}, \ldots, p_{K}\right)^{T}$ denotes the vector with received powers. The transmitted power is related to the received power by the log-normal distributed shadow-fading path loss coefficient $h_{i}$, i.e. $p_{i}=h_{i} \tilde{p}_{i}$, with $\tilde{p}_{i}$ as transmitted power of the $i$ th user. In the following, we refer our evaluation to the received power.

The SINR of user $i$ at the output of the linear detector can be expressed by

$$
S I N R_{i}=\frac{\psi_{i, i} p_{i}}{\sum_{k \neq i}^{K} \psi_{i, k} p_{k}+\sigma_{n}^{2}} .
$$

The element $\psi_{i, k}$ describes the cross-coupling between the $i$-th user and $k$-th interferer after linear filtering and can be collected in $K \times K$ matrix $\boldsymbol{\Psi}$. The matrix $\boldsymbol{\Psi}$ includes all system characteristics like user specific signature sequences as well as the user specific mobile radio channel profile. Note, in case of multiuser detection users are separated in a code division multiple access (CDMA) manner where the user specific signature sequences are described by the codes used in the system. UMTS applies Walsh-Hadamard codes of different length also called orthogonal variable spreading factor (OVSF) codes. With beamforming, the users get a finger print in space domain and the user specific signature is given by the array response of the beamforming array Farrokhi et al. (1998); Godara (2002). Finally, note that $\boldsymbol{\Psi}$ also depends on the linear filter applied. This filter is described by matrix $\mathbf{W}$ for the remainder of the paper, and each column of $\mathbf{W}$ represents the filter vector of one specific user. Thus, we can understand matrix $\boldsymbol{\Psi}$ as function of $\mathbf{W}$, e.g. $\boldsymbol{\Psi}=\mathrm{f}(\mathbf{W})$. With this relation and Eq. (1) we can formulate the optimization problem as

$\min _{\mathbf{W}, 0<p<P_{\max }^{L}} \sum_{i} p_{i}$ s.t. $S I N R_{i} \geq \gamma_{i}$

with $\gamma_{i}$ as required and $S I N R_{i}$ as measured signal-tointerference-plus-noise ratio. Each power is upper limited by the maximum allowable link power $P_{\max }^{L}$. The SINR requirements in Eq. (2) can be reformulated by an inequality of interference constraints Yates (1995):

$p_{i} \geq \mathbf{I}_{i}(\mathbf{p}, \mathbf{W})$

and

$\mathbf{I}_{i}(\mathbf{p}, \mathbf{W})=\frac{\gamma_{i}}{\Psi_{i i}}\left(\sum_{k \neq i}^{K} \Psi_{i k} p_{k}+\sigma_{n}^{2}\right)$ 
describes the effective interference seen by the $i$ th user. It is important to note that the problem statement in Eq. (2) is equivalent to the following one, where the optimization with respect to the linear filter is inserted in the constraint set Ulukus and Yates (1997). With Eqs. (2) and (3) the optimization problem can be rewritten in

$\min _{\mathbf{p}} \sum_{i} p_{i}$ s.t. $p_{i} \geq \min _{\mathbf{W}} \mathbf{I}_{i}(\mathbf{p}, \mathbf{W}), \quad i=1, \ldots, K$

In Eq. (5) the optimization problem is separated in an outer optimization that is defined over the power vector only, while the inner optimization assumes a fixed power vector and is defined over the filter coefficients. Since the powers are fixed over the inner minimization of the interference functions, this minimization can be re-formulated with Eqs. (1) and (4) as follows:

$\min _{\mathbf{W}} \mathbf{I}_{i}(\mathbf{p}, \mathbf{W})=\min _{\mathbf{W}} \frac{\mathbf{I}_{i}(\mathbf{p}, \mathbf{W}) p_{i}}{p_{i}}=\frac{p_{i} \cdot \gamma_{i}}{\max _{\mathbf{W}} S I N R_{i}}$.

Interpretation of Eq. (6) yields that for fixed power vectors the minimization of effective interference with respect to the filter matrix in the power constraints is equivalent to maximizing the SINR given by Eq. (1). Hence, the MMSE filter is the optimum linear filter solution of the optimization problem Eq. (2) Farrokhi et al. (1998); Yener et al. (2001); Visotsky and Madhow (1999).

In Foschini and Mijanic (1993) it has been recognized for single antenna reception and simple matched-filtering in the code domain $(\mathbf{W}=\mathbf{I})$ that the optimal solution can also be approached iteratively:

$p_{i}(l)=\frac{\gamma_{i}}{S I N R_{i}(l-1)} \cdot p_{i}(l-1)$

for $i=1, \ldots, K$. Parameter $l$ denotes the $l$-th iteration step.

Based on the the concept of standard interference function introduced in Yates (1995) it has been shown in Ulukus and Yates (1997) that the optimal solution to the power control problem can still be found by an iteration when the matrix filter $\mathbf{W}$ depends on $\mathbf{p}$ as it is the case for MMSE. In this case the optimal solution is given by a two-stage iterative algorithm, where within each power update Eq. (7) the MMSE filter has to be re-calculated. Again, the above statement is valid for MMSE multiuser detection as well as MMSE based beamforming Farrokhi et al. (1998); Ulukus and Yates (1997); Yener et al. (2001); Dekorsy and Brueck (2003).

\subsection{Load control}

Beside link specific power restrictions in Eq. (2) it is of utmost importance in UMTS to keep the air interface load under predefined thresholds, especially in order to guarantee the needed QoS requirements of already established calls. Load control for UMTS dedicated channels composes admission and congestion control. While the first one controls the set up of new calls the latter one is responsible for already established calls. In UMTS the cell specific load can be monitored by measuring the totally radiated or transmitted power of a sector utilizing the power rise in downlink and noise rise in uplink Holma and Toskala (2000); Mueckenheim and Bernhard (2001). The power rise $P R$ is defined as a sector's total transmit power $P^{t o t}$ over the power $P_{\text {pilot }}$ dedicated to the primary common pilot channel (P-CPICH):

$P R:=\frac{P^{t o t}}{P_{\text {Pilot }}}$,

where the total power $P^{t o t}$ is composed of the sum of all user powers $p_{i}$ and the P-CPICH power ${ }^{2}$. Note that the P-CPICH consumes $10 \%$ of the maximum transmitted sector power. If Secondary Common Pilot Channels (S-CPICH) exist as used for the fixed beam approach Schacht et al. (2003), they also contribute to $P^{\text {tot }}$.

For the uplink, the noise rise is defined as:

$N R:=\frac{I_{0}}{I_{t h}}$.

Here $I_{0}$ denotes the totally received wide band power at the antenna connector including user signal, interference as well as noise. $I_{t h}$ is the thermal noise power in the $3.84 \mathrm{MHz}$ frequency band. A new call will be established if both the $N R$ as well $P R$ remains lower than a maximum allowable threshold. This decision is due to admission control. Moreover, congestion control drops an active subscriber if the $N R$ or $P R$ exceeds a given congestion threshold. A typical value for admission control is $6 \mathrm{~dB}$ while for congestion control a typical value is $10 \mathrm{~dB}$ Mueckenheim and Bernhard (2001).

\section{UMTS downlink beamforming}

\subsection{Beamforming concepts}

The concepts to be investigated are 3-sectorization as reference, fixed beam forming ( $\mathrm{FxB}$ ), and adaptive (userspecific) beamforming (ABF). For the remainder of the paper we assume a uniform linear array (ULA) at the center of each cell composed of four antenna elements with halfwavelength spacing. Since UMTS is designed with low data rate signalling feedback channels to obtain high bandwidth efficiency Holma and Toskala (2000), neither the instantaneous channel coefficients nor array response vectors are known at the base station for downlink beamforming. In this paper we assume that the beamforming weights per user are computed in advance by exploiting the long-term spatial characteristics of the uplink channel Godara (2002); Czylwik and Dekorsy (2001). This approach is especially valid when considering practicable beamforming solutions suitable for UMTS applications. However, it requires the same data mix in uplink and downlink. With this assumption of pre-computed, and thus, fixed beamforming weights in the downlink, we only have to control the power.

\footnotetext{
${ }^{2}$ Further downlink channels are not considered herein.
} 
Table 1. Traffic mix.

\begin{tabular}{ccc}
\hline $12.2 \mathrm{kbit} / \mathrm{s}$ & $64.4 \mathrm{kbit} / \mathrm{s}$ & $384 \mathrm{kbit} / \mathrm{s}$ \\
\hline $50 \%$ & $40 \%$ & $10 \%$ \\
\hline
\end{tabular}

Table 2. SINR requirements.

\begin{tabular}{ccc}
\hline Data rate & Downlink SINR requ. & BLER in \% \\
\hline $12.2 \mathrm{kbit} / \mathrm{s}$ & $7.4 \mathrm{~dB}$ & 0.7 \\
$64 \mathrm{kbit} / \mathrm{s}$ & $4.2 \mathrm{~dB}$ & 5 \\
$384 \mathrm{kbit} / \mathrm{s}$ & $2.7 \mathrm{~dB}$ & 5 \\
\hline
\end{tabular}

\subsubsection{Adaptive beamforming}

The adaptive beamforming strategy investigated is to maximize the received uplink signal power while maintaining the total interference consisting of intra-, inter-cell interference, and thermal noise. The optimization yields a generalized eigenvalue problem where the optimum beamforming solution $\mathbf{w}_{k}^{o p t}$ per user $k$ is the generalized eigenvector corresponding to the largest eigenvalue Godara (2002). Note, UMTS suffers strong pilot transmission in the downlink due to powerful P-CPICH transmission. We consider the strong pilot transmission by diagonal loading concepts. Thus, we simply add P-CPICH signal power (weighted by the uplink channel pathloss) on the diagonal elements of the given uplink interference covariance matrix. This diagonal loading weaks nulling of interferers and transfers the beamforming strategy to maximization of the signal-to-noise ratio (SNR). Results not shown herein indicate maximization of SNR to be the preferable adaptive beamforming solution.

A problem $\mathrm{ABF}$ has to cope with is the degradation of the channel estimation which can no longer be based on the P-CPICH due to user specific antenna patterns, but has to use the pilot bit sequence on the dedicated physical control channel (DPCCH). Since this sequence is not continuously and sent out with less power than the P-CPICH we assume a worst case degradation of $3 \mathrm{~dB} 3 \mathrm{GPP}$ (2002).

\subsubsection{Fixed beamforming}

For fixed beamforming the ULA is used to preform a number of fixed beams which cover the area of a standard $120^{\circ}$-sector 3GPP (2002). Assignment of users to beams is based on the maximum received uplink power per beam. In order to allow proper channel estimation and beam identification, an additional S-CPICH is established per beam. Their powers are adjusted to provide the same SINR as on the P-CPICH.

To accomplish downlink beamforming the characteristics of the individual antenna elements have to be considered Czylwik and Dekorsy (2001). Here, we apply antenna diagrams with squared cosine shapes Czylwik and Dekorsy (2001); Schacht et al. (2002). If not declared differently, a $3 \mathrm{~dB}$ angular aperture of $120^{\circ}$ is used.
Table 3. SINR gains of beamforming concepts.

\begin{tabular}{cccc}
\hline & Standard Sectorization & FxB & ABF \\
\hline SINR in $\mathrm{dB}$ & 0 & 4 & 3 \\
\hline
\end{tabular}

Table 4. Parameters.

\begin{tabular}{cc}
\hline Path loss & COST-HATA \\
\hline Shadow fading stand. dev. & $8 \mathrm{~dB}$ \\
Max. base station power & $43 \mathrm{dBm}$ \\
Cell radius & $1000 \mathrm{~m}$ \\
Size active set & 4 \\
Hand-Over Add-margin & $4 \mathrm{~dB}$ \\
Hand-Over Drop-margin & $6 \mathrm{~dB}$ \\
PC step size & $1 \mathrm{~dB}$ \\
PC error, stand. dev. & $1.5 \mathrm{~dB}$
\end{tabular}

\subsection{System level investigations}

Within this section we work out the performance improvement by smart antennas when combined with UMTS specific radio resource management (RRM) algorithms as power control, handover, and load control Holma and Toskala (2000). It should be stressed that all RRM algorithms investigated are practicable for UMTS. Thus, the main objective here is to work out the performance of beamforming methods applicable for UMTS under the constraint of RRM algorithms and their corresponding parameter settings. We also consider heterogeneous traffic with different data rates listed in Table 1.

The investigation approach was as follows. In a first step, we worked out required SINR values for different UMTS services by detailed physical layer simulations. Note that for UMTS the QoS is expressed by a block error rate (BLER) that corresponds to an SINR on the Dedicated Physical Data Channel (DPDCH). Table 2 lists these required SINRs and their corresponding BLERs.

In order to include spatially formed interference (inter- as well as intra-cell interference) intensive system level simulations were carried out for the beamforming concepts of interest. Table 3 summarizes the gain in terms of an averaged SINR per user after RAKE reception for mixed traffic scenarios. Since fixed beamforming significantly decreases with larger angular spread Schacht et al. (2002) we focus here on an angular spread of $1^{\circ}$. Finally, with the results summarized in Table 3 dynamic system level simulations were carried out using a system level simulator including all RRM algorithms mentioned above. The main simulation parameters are listed in Table 4. Note that a UMTS applicable power control algorithm with $1 \mathrm{~dB}$ stepsize and log-normal distributed power control error with $1.5 \mathrm{~dB}$ standard deviation is used.

Aside from the reduction of the totally emitted power, another important parameter to be investigated is the call-errorrate (CER). The CER is the sum of blocking as well as drop- 


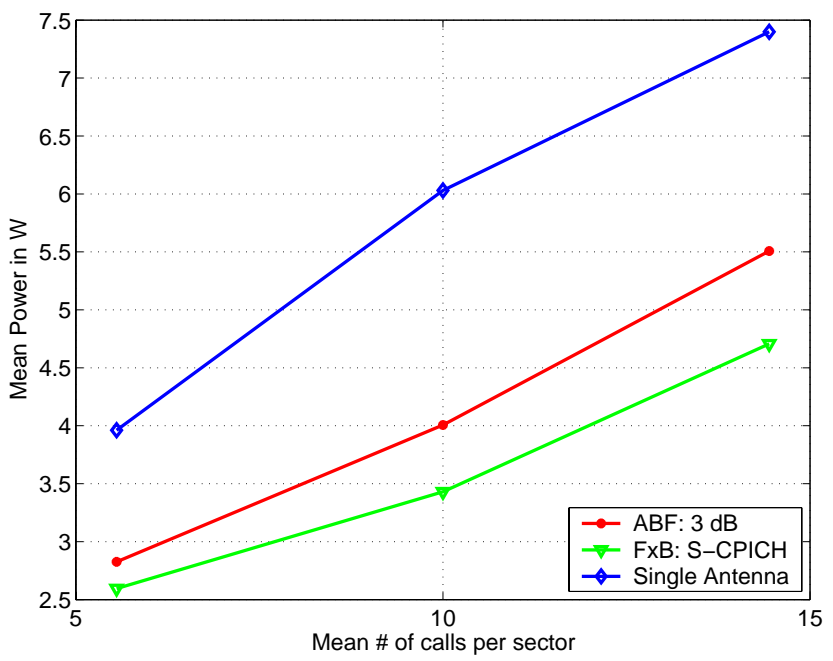

Fig. 1. Mean power vs. load per sector, ULA, $M=4$ elements.

ping rate of the network. The blocking rate itself is defined by the ratio of the number of blocked calls over the total number of calls. Same holds for the dropping rate, respectively. A call is blocked if at least one of the following events occur: SINR of the P-CPICH is lower than the required one or if the actual power rise is higher than the maximum allowable limit (admission control). We assumed a power rise of $6 \mathrm{~dB}$ for blocking. A call drops if at least one of the following events occur: The transmitted power of the user reaches its maximum and the DPDCH (Dedicated Physical Data Channel) can not meet the SINR requirement, or the power rise exceeds its upper limit. Here, the latter aspect defines the congestion control Mueckenheim and Bernhard (2001) and the upper limit is set to $10 \mathrm{~dB}$. To assess the reduction of radiated power, we used the mean power of a sector. Fig. 1 displays the mean power of a sector versus the network load for the different antenna concepts. It is clearly indicated that the beamforming concepts reduce the emitted power by the base stations compared to the reference of 3 sectorization. For adaptive beamforming we find a power reduction of about $2 \mathrm{~dB}$ and for fixed beam switching $2.3 \mathrm{~dB}$. The reason for fixed beam to be superior can be explained by (1) an angular spread of $1^{\circ}$ that is much more narrow than the beamwidth of a fixed beam, and (2) ABF suffers a high SINR loss of $3 \mathrm{~dB}$ due to weak channel estimation on the DPCCH. The results clearly show the potential of beamforming concepts to reduce the emission of UMTS base stations. In addition to emission reduction, beamforming concepts can tremendously improve system capacity. Figure 2 shows the corresponding call-error-rate for the different antenna concepts versus the load per sector. For UMTS services a maximum CER of $2 \%$ is often demanded by service providers. The results in Fig. 2 clearly demonstrate that a network with ordinary sectorization is not capable of meeting that restriction for the traffic mix assumed and 10 calls per sector in average. In contrast beamforming concepts can handle the traffic. For user specific beamforming we observed a CER of $1 \%$, while

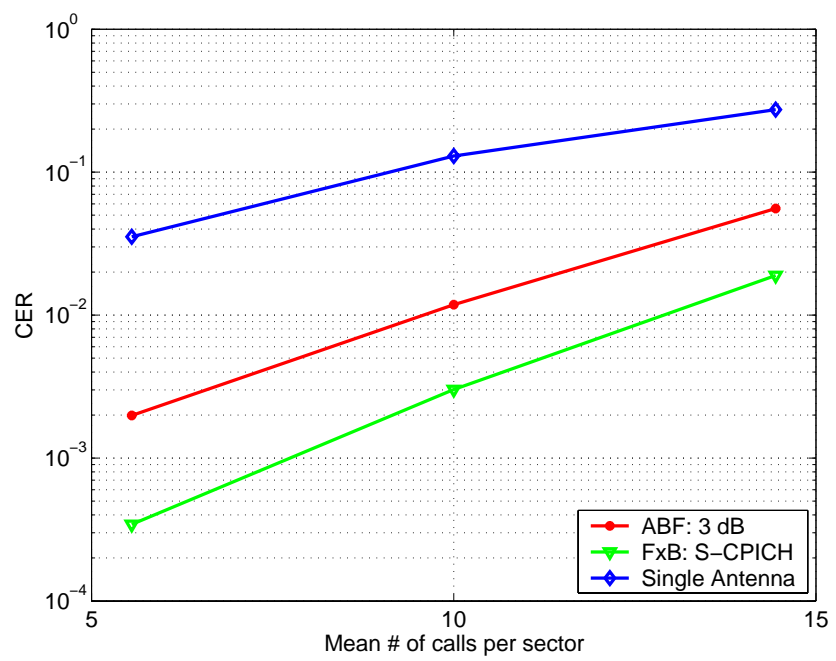

Fig. 2. CER vs. load per sector, ULA, $M=4$ elements.

for fixed beam switching the CER is of app. 0.3\%. In summary, applying beamforming concepts for UMTS downlink transmission leads to a reduction of an averaged totally transmitted power per base station of approx. 2-2.5 dB with an additional reduced call-error-rate.

\section{Multiuser detection for UMTS}

Besides beamforming multiuser detection represents another approach to cope with high co-channel interference of CDMA based systems. In this paper, we consider MMSE multiuser block detection for asynchronous mixed service UMTS networks. The basic modulation scheme of the UMTS uplink is BPSK whereby the user data are mapped onto antipodal in-phase bits, while control (pilot, power control) information are mapped onto antipodal quadraturephase bits Holma and Toskala (2000). The control and data bit sequences are spread by mutually orthogonal Walsh codes (Orthogonal Variable Spreading Factor (OVSF) codes). The resulting spread time-slot signal is scrambled by a mobilespecific scrambling code. UMTS offers to apply long as well as short scrambling codes. Short scrambling codes with length of 256 chips (one control symbol) allow significant reduction in complexity and are assumed in this paper. Multiuser detection in asynchronous transmission can only be performed optimally by considering a signal block of appropriate length Verdu (1998), here denoted by $L$. Depending on its data rate user $k$ transmits $N(k)$ symbols within this period of length $L$. As an example, a voice user of rate $12.2 \mathrm{kbit} / \mathrm{s}$ applies Walsh codes of length 64 resulting in $N(k)=40$ symbols per block of length $L=2560$ chips. Moreover, in this paper inter-cell interference is assumed to be proportional to the power in the cell of interest and is modelled by the intercell interference factor $f$ Holma and Toskala (2000). This model ensures coupling between sectors of a fully deployed sectorized network. 


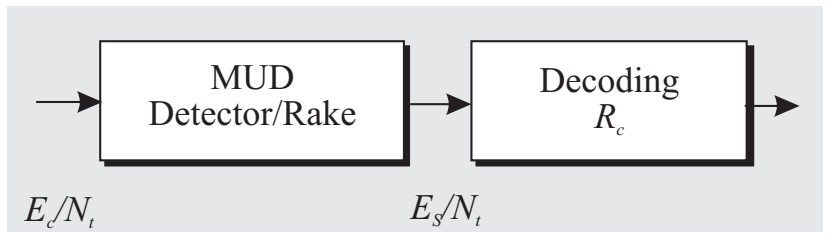

Fig. 3. Basic signal processing diagram of DPDCH.

\section{SINR operating points in UMTS}

This section gives some essential comments on the SINR operating points of multiuser detectors. The link quality in UMTS is defined by the BLER that has to be ensured for the service offered. This BLER corresponds to an averaged SINR. This averaged SINR requirement is measured on the $\mathrm{DPCCH}$, the inner loop (fast) power control has to adjust the transmitted powers in order to ensure the link quality. Note that directly associated with the SINR on the DPCCH is an SINR on the DPDCH. It is common to express the SINR requirement on the DPDCH in terms of energy per information bit versus overall interference, often denoted by $E_{b} / N_{t}$ Holma and Toskala (2000). Be aware that this $E_{b} / N_{t}$ requirement strongly depends on the environment (PedA-, VehA-channel, etc.), on receiver performance as channel estimation algorithms as well as the service offered. Figure 3 shows the basic signal processing diagram of the DPDCH and the corresponding SINR requirements. At the input of the decoder the SINR can be expressed in terms of energy per symbol $E_{S}$ while before MUD detection we have the energy per chip $E_{c}$.

These different SINRs are given in linear domain by:

$\frac{E_{s}}{N_{t}}=\frac{E_{b}}{N_{t}} R_{c}$, with $R_{c}<1$,

where $R_{c}$ is the channel coding rate. Moreover, for simple Rake detection we have $E_{c} / N_{t}=E_{s} / N_{t} 1 / S F$ with SF as spreading factor of the service. Parameter $N_{t}$ takes into account the overall interference seen by the user signal before decoding. If multiuser detection is applied the MUD detector operates on symbol energy basis as indicated by Fig. 3 . Hence, the operating point for the MUD detector is given by the $E_{s} / N_{t}$ value that has to be ensured by the inner power control. Table 5 lists some typical $E_{s} / N_{t}$ values for different uplink UMTS services in single rate systems. Under inclusion of these UMTS constraints we obviously obtain very low $E_{S} / N_{t}$ values, and, hence, very low operating points of the multiuser detectors. Note that these SINR operating points represent the former introduced SINR requirement denoted by $\gamma=E_{s} / N_{t}$. In addition, Table 5 lists required SINR values $E_{c} / N_{t}$ before despreading as well. It is has been shown above that the joint optimization problem Eq. (2) ends up in a MMSE filter solution. But the problem arising with linear MUD is that at low SNR operating points simple RAKE detection (single user matched filtering) converges to the optimum MMSE solution. This is because of

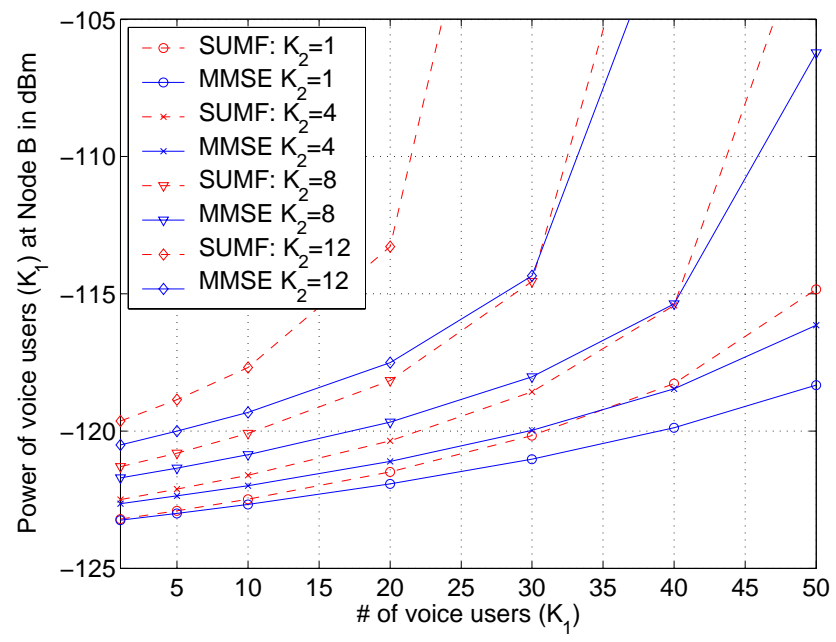

Fig. 4. Power of one voice user, $f=0.83$, and $L=512$.

the trade-off between cancellation of intra-cell interference and the drawback of noise enhancement that is inherent in linear filtering in general. This property of linear MUD was intensively worked out in the past and is well known in literature, e.g. Verdu (1998).

\subsection{System-level investigations}

In the following we show results of linear MMSE block detection in asynchronous mixed-service UMTS networks. For asynchronous transmission we have to consider a signal block of appropriate length that is here chosen to be $L=512$. Within this paper, the service mix composes voice with rate $12.2 \mathrm{kbit} / \mathrm{s}$ (service 1) and data transmission with rate 64 $\mathrm{kbit} / \mathrm{s}$ (service 2), refer to Table 5. The relative user delays are up to $\tau_{\max }=30$ chips to take into account asynchronous transmission. We focus on 6-sector networks with cell radius of $1000 \mathrm{~m}$, and the fractional cell loading factor is chosen to be $f=0.83$ Holma and Toskala (2000). Thus, the amount of inter-cell interference a user has to deal with is of $83 \%$ of the intra-cell interference the user exposes to. Finally, $K_{1}$ and $K_{2}$ denotes the load of the voice and data service, respectively, which will both be varied in our investigations in the following. Finally SUMF performance is used as reference throughout the investigations.

Figure 4 shows the required power at the base station receiver of one voice user for different service mixes. In general, we observe that the higher the load is the more power can be saved by MMSE compared to SUMF. Especially an increase of data users results in higher MMSE performance gains. As an example, for $K_{1}=20$ and $K_{2}=1$ MMSE reduces the power in the order of $1 \mathrm{~dB}$ only, while for same number of voice but higher number $K_{2}=12$ of data users MMSE results in 3-4 dB less power. Note that voice users have less impact on the MMSE performance gain due their significant lower interference contribution. Although the spreading factor of the data service is four times the voice spreading factor, the SINR requirement of the data service is more relaxed 
Table 5. Required SINR values for different UMTS services.

\begin{tabular}{ccccccc}
\hline Data Rate [kbit/s] & BLER [\%] & SF & $R_{c}$ & $E_{b} / N_{t}[\mathrm{~dB}]$ & $\gamma=E_{s} / N_{t}[\mathrm{~dB}]$ & $E_{c} / N_{t}[\mathrm{~dB}]$ \\
\hline 12.2 & 0.7 & 64 & $\frac{1}{4.9}$ & 4.5 & -2.4 & -20.46 \\
64 & 5 & 16 & $\frac{1}{3.75}$ & 2 & -3.7 & -15.7 \\
\hline
\end{tabular}

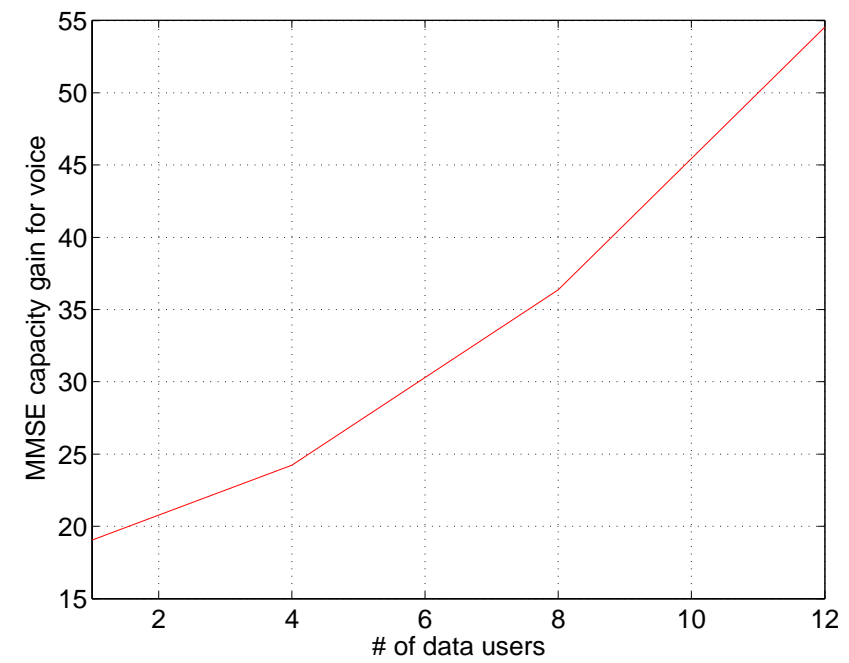

Fig. 5. MMSE capacity gain for voice, $f=0.83$ and $L=512$.

yielding to approximately 3-4 dB more power consumption of the data service to meet its requirement, refer to Table 5. Compared to SUMF the MMSE results in almost identical power reduction for both services which is in the range of 2-3 dB measured for service loads on a noise rise level of 6 $\mathrm{dB}$, refer to Fig. 6. A noise rise level of $6 \mathrm{~dB}$ is typical for quoting system capacity.

Also for this noise rise, Fig. 5 depicts the MMSE capacity gains for voice versus data load. The results indicate the higher the data service load the higher the gain for MMSE in terms of voice capacity. But, due to the very low SNR operating points we obtain fairly modest MMSE gains only. As an example, for $K_{2}=1$ (almost single rate), we observe a capacity increase of voice users in the order of $18 \%$ only, while for $K_{2}=8$ we obtain an MMSE gain of $36 \%$. Even for very high data loads the capacity stays below $60 \%$ (note that for single rate data system, i.e. no voice users, the maximum number of servable data users for SUMF at $N R=6 \mathrm{~dB}$ is 14 users, refer to Dekorsy and Brueck (2003).

Finally, Fig. 6 shows the maximum service loads that can be served by the two detectors for noise rise levels $6 \mathrm{~dB}$ and $8 \mathrm{~dB}$. The graphs can be interpreted as "rate-regions" for the given service mix taken of data (64 kbps) and voice (12 kbps) subject to noise rise threshold $6 \mathrm{~dB}$ or $8 \mathrm{~dB}$, respectively. Figure 9 clearly indicates how MMSE extends the "rate region".

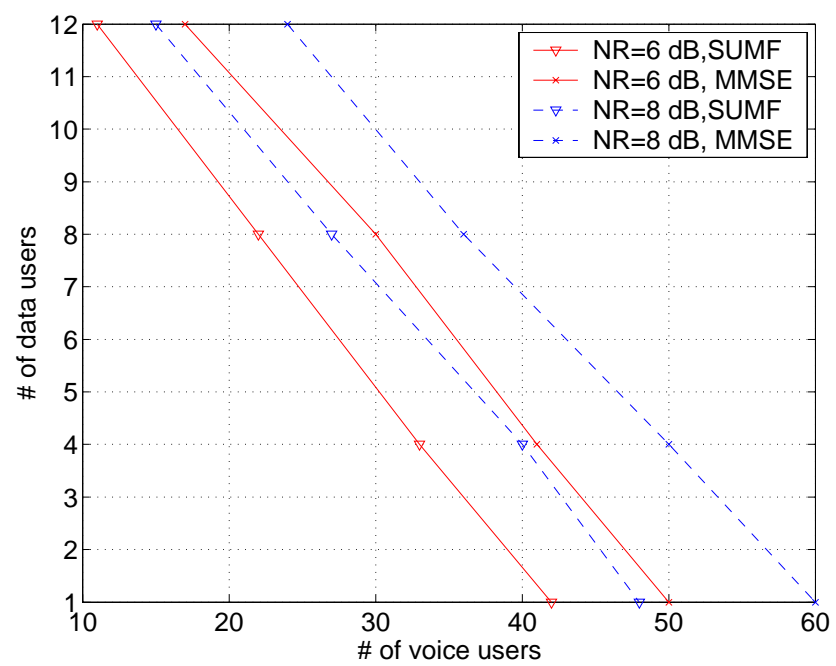

Fig. 6. Maximum service mix load for different noise rise levels.

\section{Conclusion}

This paper investigated the applicability of fixed and adaptive beamforming concepts as well as multiuser detection for mixed service UMTS networks. Special interest was given to the interaction of these promising techniques with UMTS power- and load control strategies. For beamforming the focus was on downlink, while multiuser detection was applied in the uplink direction. With the application of smart antennas (ULA with 4 elements) we observed a tremendous reduction of the call-error-rate. On top of higher quality the radiated base station power can be reduced by at least $2 \mathrm{~dB}$. In the uplink, multiuser detection performance enhancements are severely limited by very low SINR operating points. With this restriction, MMSE error block detection shows fairly modest capacity gains. Nevertheless, MMSE results in fair to high power reduction at the mobile station in the range of 2-3 dB. 


\section{References}

Czylwik, A. and Dekorsy, A.: System Level Simulations for Downlink Beamforming with Different Array Topologies, GLOBECOM, San Antonio, USA, 3222-3226, 2001.

Dekorsy, A. and Brueck, S.: On System Capacity and Coverage Improvements by Linear MUD for UMTS, VTCS, Jeju, South Korea, 2003.

Farrokhi, R. F., Liu, K. J. R., and Tassiulas, L.: Transmit Beamforming and Power Control for Cellular Wireless Systems, JSAC, 16, 8, 1437-1449, 1998.

Foschini, G. J. and Miljanic, Z.: A simple distributed autonomous power control algorithm and its convergence, ITVT, 42, 641646, 1993.

3GPP, Technical Specification Group Radio Access Network: Proposal for user specific beamforming for UTRA FDD, Document for Discussion and Decision, TSGR4-17, ETSI, 2001.

Godara, L. C.: Handbook of Antennas in Wireless Communications, CRC Press, 0-8493-0124-6, 2002.

Holma, H. and Toskala, A.: WCDMA for UMTS, John Wiley \& Sons, LTD., Chichester, England, 2000.
Mueckenheim, J. and Bernhard, U.: A framework for load control in 3rd generation CDMA networks, GLOBECOM, San Antonio, USA, 2001.

Schacht, M., Dekorsy, A., and Jung, P.: System Capacity from UMTS Smart Antenna Concepts, VTCF, Orlando, USA, 2003.

Schacht, M., Dekorsy, A., and Jung, P.: Downlink Beamforming Concepts in UTRA FDD, Kleinheubacher Tagung, Kleinheubach, Germany, 2002.

Ulukus, S. and Yates, R. D.: Adaptive Power Control and MMSE Interference Suppression, Wireless Networks, Special Issue on Multiuser-Detection in Wireless Communications, 1997.

Verdu, S.: Multiuser Detection, Cambridge University Press, UK, 1998.

Visotsky, E. and Madhow, U.: Optimum Beamforming Using Transmit Antenna Arrays, VTC, 851-856, 1, 1999.

Yates, R. D.: A Framework for Uplink Power Control in Cellular Radio Systems, JSAC, 13, 7, 1341-1347, 1995.

Yener, A. and Yates, R. D., and Ulukus, S.: Interference Management for CDMA Systems Through Power Control, Multiuser Detection, and Beamforming, ITC, 49, 7, 1227-1239, 2001. 